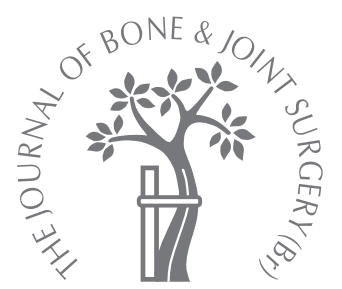

T. Shisha,

S. Kiss,

K. Pap,

H. Simpson,

G. Szöke

From Semmelweis

University, Budapest, Hungary

\title{
Relative ability of young and mature muscles to respond to limb lengthening
}

The response of the muscle is critical in determining the functional outcome of limb lengthening. We hypothesised that muscle response would vary with age and therefore studied the response of the muscles during tibial lengthening in ten young and ten mature rabbits. A bromodeoxyuridine technique was used to identify the dividing cells.

The young rabbits demonstrated a significantly greater proliferative response to the distraction stimulus than the mature ones. This was particularly pronounced at the myotendinous junction, but was also evident within the muscle belly.

Younger muscle adapted better to lengthening, suggesting that in patients in whom a large degree of muscle lengthening is required it may be beneficial to carry out this procedure when they are young, in order to achieve the optimal functional result.

The response of striated muscle during limb lengthening is a major determinant of functional outcome. Major complications such as contractures, subluxations or dislocations can result from the failure of muscle-tendon units to attain an adequate length. ${ }^{1-3}$ Although there have been some accounts of the role of the myotendinous junction in the longitudinal growth of muscles during limb lengthening, 4,5 there is no information available on its proliferative activity at different ages and rates of distraction, or comparing proliferative activity at the myotendinous junction to the proliferative response within the muscle belly. ${ }^{6-10}$ The aim of this study was to provide detailed information on the proliferative activity of myogenic cells in the myotendinous junction compared with that of the muscle belly in young and mature rabbits in which the tibia had been lengthened at different rates.

\section{Materials and Methods}

Tibial osteotomy and stabilisation with an external fixator (ITEC mini distractor MCSCD-002; ITEC, Budapest, Hungary; Fig. 1) was carried out on 20 male New Zealand White Rabbits. The left hind limb was lengthened in 16 rabbits (eight young and eight mature; of which four young and four mature animals were distracted at a rate of $0.8 \mathrm{~mm} / \mathrm{day}$ and four young and four mature at a rate of $0.8 \mathrm{~mm}$ twice daily. The unoperated right hind limb served as a control in the comparison of muscle length (Table I). Four rabbits underwent osteotomy and stabilisation, but not limb lengthening (distraction rate $0 \mathrm{~mm} /$ day). The young rabbits were nine weeks old and the mature rabbits 28 weeks old, ${ }^{11}$ at which time longitudinal growth has just stopped, as in a young adult human. All of the rabbits were killed immediately after completion of the lengthening procedure. The experiment conformed to national regulations and Ethics Committee approval was obtained. Operative technique. The animals were randomly allocated to the groups. The operation was undertaken using the technique previously described. 6,11,12 After shaving and draping the left tibia, the skin and periosteum were incised longitudinally under general anaesthesia using intravenous ketamine hydrochloride and diazepam, titrated as appropriate. ${ }^{6,12}$ Four screws were placed along the longitudinal axis of the tibia and the external fixator was positioned. A transverse osteotomy of the tibia was carried out 8 to $15 \mathrm{~mm}$ distal to the tibiofibular junction, junction in the middle portion of the diaphysis (Fig. 1).

After seven days, lengthening was started in the experimental groups. The bony ends were distracted in increments of $0.8 \mathrm{~mm}$, once or twice daily, depending on the rate of lengthening. The length of the tibia was increased by $20 \%$ in every animal.

In order to label the dividing cells, the animals were injected intravenously with a thymidine analogue, bromodeoxyuridine (BrdU) (Sigma Chemical, Poole, United Kingdom) at a dose of $40 \mathrm{mg} / \mathrm{kg}$ ( $8 \mathrm{mg} / \mathrm{ml}$ saline) one hour 
Table I. The mean length (SD) of the flexor digitorum longus muscle in the different groups (in mm)

\begin{tabular}{|c|c|c|c|c|c|}
\hline & \multicolumn{2}{|c|}{ Young rabbits } & \multicolumn{2}{|c|}{ Mature rabbits } & \multirow[b]{2}{*}{$0 \mathrm{~mm} /$ day } \\
\hline & $0.8 \mathrm{~mm} /$ day & $\begin{array}{l}0.8 \mathrm{~mm} \text { twice } \\
\text { daily }\end{array}$ & $0.8 \mathrm{~mm} /$ day & $\begin{array}{l}0.8 \mathrm{~mm} \text { twice } \\
\text { daily }\end{array}$ & \\
\hline \multicolumn{6}{|l|}{ Mean length } \\
\hline Experimental side & $76.87(2.78)$ & $77.6(4.63)$ & 84.6 (8.15) & $84.7(5.6)$ & $72.6(12.4)$ \\
\hline Control side & 68.17 (1.59) & $68.4(3.58)$ & $76.9(6.07)$ & $74.4(5.3)$ & $72.3(12.2)$ \\
\hline Length increase (\%) & 12.7 & 13.4 & 10 & 13.8 & 0.4 \\
\hline
\end{tabular}

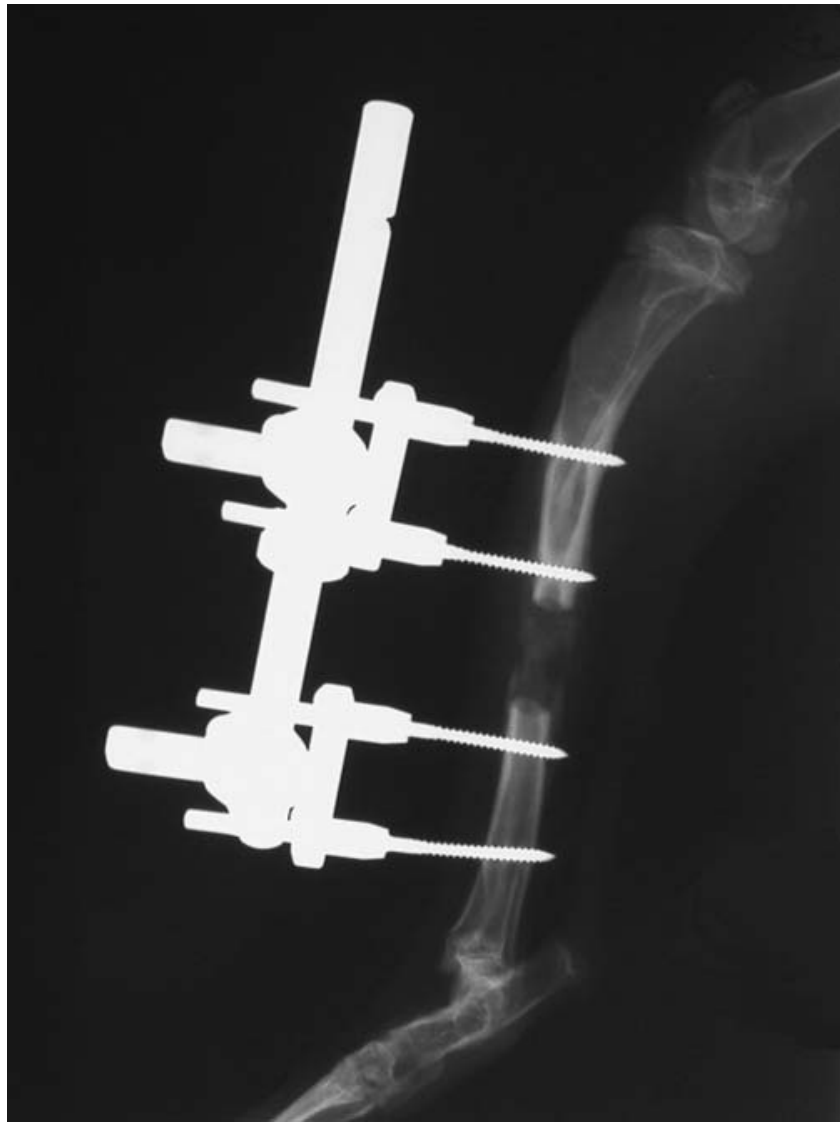

Fig. 1

Radiograph of the lengthened tibia and the external fixator.

before they were killed. BrdU competes with thymidine for incorporation into DNA. ${ }^{13}$ After incorporation into the DNA, the BrdU is stable.

Once lengthening of $20 \%$ had been achieved, the animals were killed by an overdose of intravenous barbiturate. The muscles and their tendons were carefully dissected from the bone and spread out on swabs soaked with saline at body temperature. The length of the flexor digitorum longus (FDL) muscle was measured from the proximal end of the intramuscular tendon plate to the distal end of the myotendinous junction, using a Vernier calliper (Sealey, Bury St Edmunds, United Kingdom). The muscles and tendons were processed as previously described. ${ }^{6,11,12}$ Tissues were fixed in $10 \%$ phosphate-buffered formalin for 48 hours. After fixation the samples were embedded in paraffin and $5 \mu \mathrm{m}$ longitudinal sections were cut parallel to the muscle fibres.

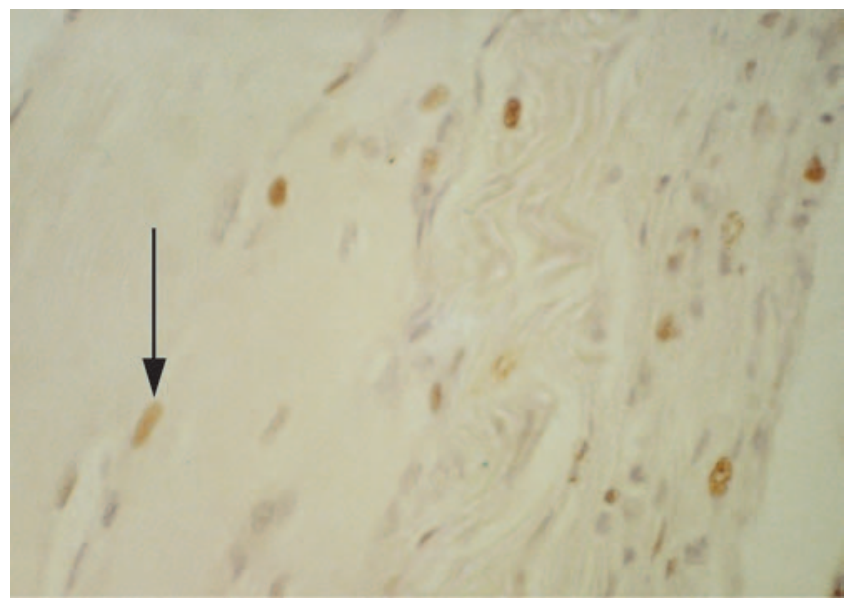

Fig. 2

Bromodeoxyuridine-labelled positive staining satellite cell in the muscle near the myotendinous junction (x 400).

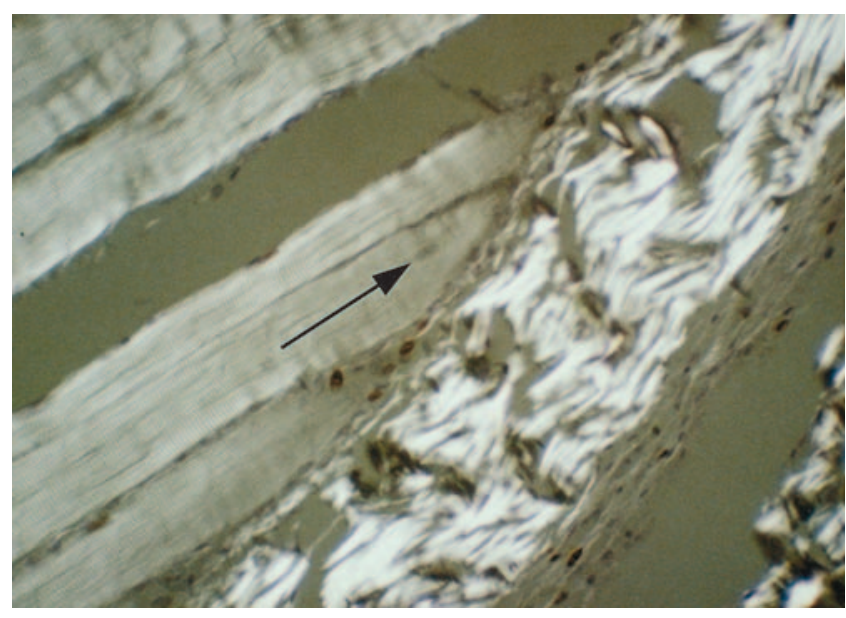

Fig. 3

Satellite cell near the myotendinous junction (polarised; $x 400$ ).

Monoclonal antibodies (Bu30a) (Sigma Chemical) highly specific to BrdU were used to detect its presence in the cell nuclei, as described by Magaud et al. ${ }^{14}$ For counterstaining of the negative nuclei, a weak Mayer's haematoxylin stain was used which was sufficiently strong to demonstrate the negative nuclei but not strong enough to mask weak positive reactions (Figs 2 and 3). Two serial sections of small bowel served as positive controls and two serial sections of 


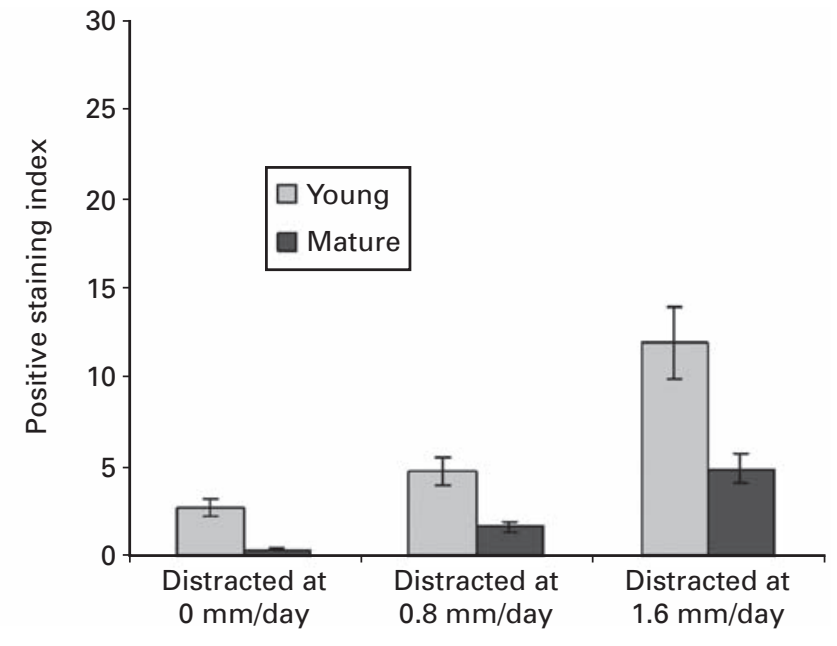

Fig. 4

The positive staining index in the muscle belly of distracted and dis tracted at $0 \mathrm{~mm} /$ day muscles (mean and $95 \%$ confidence interval).

Table II. The mean positive staining index (\%) in the muscle belly and in the myotendinous junction in the different groups. The p-value is calculated for the difference between young and mature rabbits

\begin{tabular}{lcccc}
\hline & Young (SD) & Mature (SD) & p-value \\
\hline Musculotendinous junction & & & & \\
$0 \mathrm{~mm}$ /day & $8.6(3.19)$ & $1.92(0.41)$ & $<0.005$ \\
$0.8 \mathrm{~mm}$ /day & 14 & $(4.92)$ & $4.67(1.87)$ & $<0.007$ \\
$0.8 \mathrm{~mm}$ twice daily & $28.4(10.3)$ & 8.6 & $(3.07)$ & $<0.02$ \\
& & & & \\
Belly & & & & \\
$0 \mathrm{~mm} /$ day & $2.7(0.97)$ & $0.34(0.16)$ & $<0.003$ \\
$0.8 \mathrm{~mm}$ /day & $4.7(1.6)$ & $1.67(0.47)$ & $<0.01$ \\
$0.8 \mathrm{~mm}$ twice daily & $12(4.64)$ & $4.9(1.63)$ & $<0.03$ \\
\hline
\end{tabular}

liver as negative controls in each batch. The BrdU-labelled muscle nuclei were counted under the light microscope with a magnification of $x 20$, using an eyepiece. The nuclei were counted in 20 fields to determine the mean positive staining index, which is the number of positive fibre nuclei divided by the total number of muscle fibre nuclei examined $\mathrm{x} 100.6,11$ The eyepiece was placed and directed along the line of the myotendinous junction to examine a strip $50 \mu \mathrm{m}$ wide.

The Mann-Whitney U-test was used to determine significant differences between the groups (independent samples) and Wilcoxon's matched-pairs test for the comparison of experimental and control sides (dependent samples). Both of these tests are non-parametric (level of significance $\mathrm{p}<$ $0.05)$.

\section{Results}

In the group distracted at $0 \mathrm{~mm} /$ day there was no significant difference between the length of the FDL muscle on the experimental side and on the control side. The length of the muscle increased significantly in all other groups when the

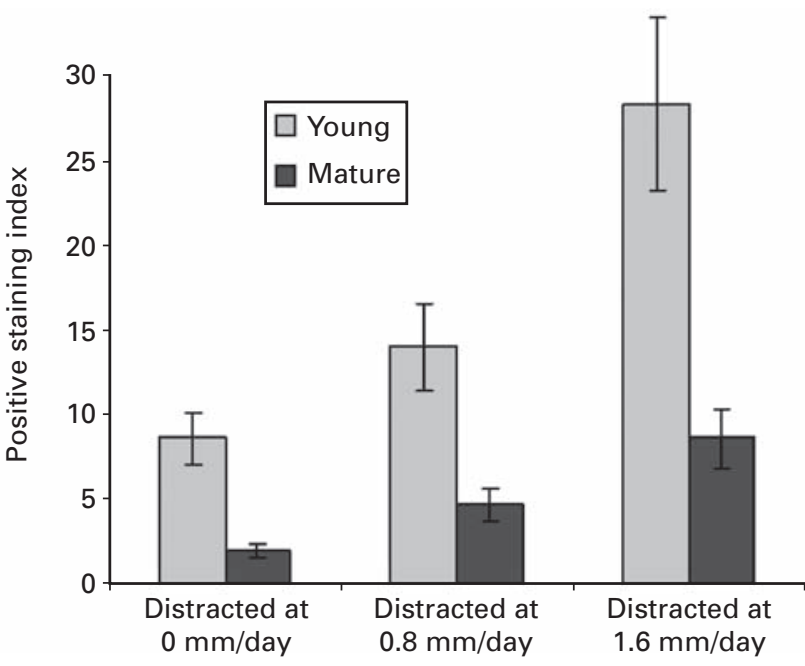

Fig. 5

The positive staining index at the myotendinous junction of distracted and distracted at $0 \mathrm{~mm} /$ day muscles (mean and $95 \%$ confidence interval).

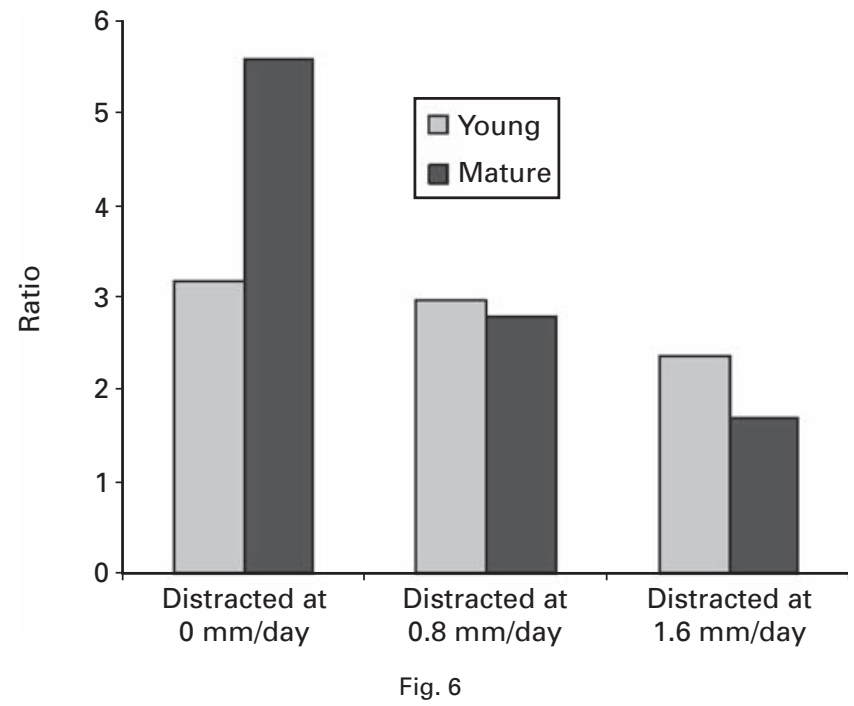

The ratio between the positive staining index of the myotendinous junction and the muscle belly. In young rabbits the dominance of the junction is less pronounced in the distracted at $0 \mathrm{~mm} /$ day muscle, but it is better preserved during distraction.

experimental side and the control side were compared (Table I).

The positive staining index of all specimens examined is shown in Table II.

In the group distracted at $0 \mathrm{~mm} /$ day there was no significant difference between the positive staining index of the experimental and control sides. In all of the lengthened limbs a significant increase of positive staining index in the muscle belly and at the myotendinous junction was observed, compared with the unlengthened control limb ( $\mathrm{p}$ $<0.01$ ). The relative increase in positive staining index in the muscle belly was greater than that observed in the myotendinous junction (Figs 4 to 6). 
At both rates of distraction, the response to lengthening in the young was far greater than in the mature animals, being over three times more at the myotendinous junction and over double in the muscle belly. On average, there were nearly six times more dividing myogenic cells in the myotendinous junction than in the muscle belly in the nondistracted muscles of mature rabbits, and a little over three times more in young rabbits (Fig. 6). In mature animals we observed a pronounced shift in the distribution of dividing cells during distraction, as although the positive staining index in the myotendinous junction remained greater than in the muscle belly, the ratio between the two decreased significantly during distraction $(\mathrm{p}<0.02$; Fig. 6). This change was the result of an increased contribution of the muscle belly to proliferation.

\section{Discussion}

Moss and LeBlond ${ }^{15}$ demonstrated that the satellite cells are responsible for the increase in the number of myonuclei which occurs during post-natal growth. These cells play an integral role in the normal development of skeletal muscle by providing a source of post-mitotic myonuclei. However, the number of satellite cells in a muscle undergoes an ordered progressive decrease over the course of post-natal life, ${ }^{16}$ and we observed significantly fewer satellite cells in the mature than in the young animals. Schumacher, Keller and Hvid ${ }^{17}$ were the first to use BrdU to label the myonuclei derived from the satellite cells, but they did not examine the myotendinous junction and were therefore unable to compare the behaviour of this site with that of the muscle belly. In order to avoid labelled fibrocytes in the close proximity of muscle fibres being counted erroneously as satellite cells, we used longitudinal sections, which helped us to distinguish these two types of cell. Our results showed an increase in the number of labelled myonuclei in the muscle belly with distraction, as observed by Schumacher et al. ${ }^{17}$ We have also demonstrated an increase in the activity in the myotendinous junction. As proliferation of satellite cells result in the formation of myofibrils, ${ }^{18}$ although the myotendinous junction is a key site in the longitudinal growth of striated muscles, the previous view that the growth of muscle fibres occurs exclusively at the end of fibres should be revised. ${ }^{4,18,19}$

Simpson et $\mathrm{al}^{6}$ found that limbs of skeletally-mature animals which were lengthened at a rapid rate developed contractures. However, even at rapid rates of distraction, these authors demonstrated that new sarcomeres were added with the creation of new muscle tissue. Our results support these findings.

It has previously been suggested that muscle contractures associated with distraction osteogenesis may be a function of age. ${ }^{20}$ We agree with this, and have clearly demonstrated that younger muscle has a better growth potential during limb lengthening. This should be borne in mind when planning programmes where a large amount of lengthening is required.

No benefits in any form have been received or will be received from a commercial party related directly or indirectly to the subject of this article.

\section{References}

1. Paley D. Problems, obstacles and complications of limb lengthening by the llizarov technique. Clin Orthop 1990;250:81-104.

2. Jones DC, Moseley CF. Subluxation of the knee as a complication of femoral lengthening by the Wagner technique. J Bone Joint Surg [Br] 1985;67-B:33-5

3. Williams P, Simpson H, Kyberd P, Kenwright J, Goldspink G. Effect of rate of distraction on loss of range of joint movement, muscle stiffness, and intramuscular connective tissue content during surgical limb lengthening: a study in the rabbit. Anat $\operatorname{Rec}$ 1999;255:78-83

4. Dix DJ, Eisenberg BR. Myosin mRNA accumulation and myofibrillogenesis at the myotendinous junction of stretched muscles. J Cell Biol 1990;111:1885-94.

5. Goldspink G, Williams $\mathbf{P}$, Simpson H. Gene expression in response to muscle length and mass. Clin Orthop 2002;403(Suppl):146-52.

6. Simpson AH, Williams PE, Kyberd P, Goldspink G, Kenwright J. The response of muscle to leg lengthening. J Bone Joint Surg [Br] 1995;77-B:630-6.

7. Kyberd PJ, Williams PE, Simpson AHRW, Goldspink G, Kenwright J. The response of the mammalian muscle to distraction: a study in the rabbit. Procs Inst Mech Eng H, J Eng Med 1994;208:111-18.

8. McKoy G, Ashley W, Mandler J, et al. Expression of insulin growth factor-1 splice variants and structural genes in rabbit skeletal muscle induced by stretch and stimulation. J Physiol 1999;516(Pt 2):583-92.

9. Allen DL, Monke SR, Talmadge RJ, Roy RR, Edgerton VR. Plasticity of mononuclear number in hypertrophied and atrophied mammalian skeletal muscle fibers. $J$ Appl Physiol 1995;78:1969-76.

10. De Deyne PG. Lengthening of muscle during distraction osteogenesis. Clin Orthop 2002;403(Suppl 1):171-7

11. Szöke G, Lee SH, Simpson AH, Prescott J. Response of the tendon during limb lengthening. J Bone Joint Surg [Br] 2005;87-B:583-7.

12. Apte SS. Immunohistochemical studies of cell proliferation in skeletal tissues [the sis]. Oxford: University of Oxford Press, 1990:21-47.

13. Goz B. The effects of incorporation of 5 -halogenated deoxyuridines into DNA of eukaryotic cells. Pharmcol Rev 1978;29:249-72.

14. Magaud JP, Sargent I, Clarke PJ, et al. Double immunocytochemical labeling of cell and tissue samples with monoclonal anti-bromodeoxyuridine. J Histochem Cytochem 1989;37:1517-27.

15. Moss FB, LeBlond CP. Satellite cells as the source of nuclei in muscles growing rats. Anat Rec 1971;170:421-35

16. Campion DR. The muscle satellite cell: a review. Int Rev Cytol 1984;87:225-51

17. Schumacher B, Keller J, Hvid I. Distraction effects on muscle: leg lengthening studied in rabbits. Acta Orthop Scand 1994;65:647-50.

18. Caiozzo VJ, Utkan A, Chou $\mathbf{R}$, et al. Effects of distraction on muscle length: mechanisms involved in sarcomerogenesis. Clin Orthop 2002;403(Suppl):133-45.

19. Williams PE, Goldspink G. The effect of immobilization on the longitudinal growth of striated muscle fibers. J Anat 1973;116(Pt 1):45-55.

20. Hayatsu K, De Deyne PG. Muscle adaptation during distraction osteogenesis in skeletally immature and mature rabbits. J Orthop Res 2001:19:897-905. 